\title{
An Interesting Case Presentation of Burkitt Lymphoma Presenting with Lactic Acidosis and Thrombocytopenia
}

\author{
Abdul Miah* \\ Division of Medical Oncology, Department of Internal Medicine, The Ohio State University College of Medicine, USA
}

*Corresponding author: Abdul Miah, Division of Medical Oncology, Department of Internal Medicine, The Ohio State University College of Medicine, Columbus, Ohio, USA, E-mail: abdul.miah@osumc.edu

Received: 03 Apr, 2020 | Accepted: 27 Apr, 2020 | Published: 01 May, 2020

Citation: Miah A (2020) An Interesting Case Presentation of Burkitt Lymphoma Presenting with Lactic Acidosis and Thrombocytopenia. J Clin Case Stu 5(2): dx.doi.org/10.16966/2471-4925.201

Copyright: (C) 2020 Miah A. This is an open-access article distributed under the terms of the Creative Commons Attribution License, which permits unrestricted use, distribution, and reproduction in any medium, provided the original author and source are credited.

\section{Abstract}

Burkitt Lymphoma (BL) is a very aggressive B-cell lymphoma caused by the translocation and subsequent dysregulation of the c-Myc gene on chromosome 8. There are 3 distinct clinical forms of Burkitt Lymphoma: endemic (African), sporadic (non-endemic), and immunodeficiency associated. Although these forms differ in their clinical presentation and their epidemiology, they share the same aggressive clinic behavior and are histological identical. This case presentation reports a rare case of sporadic form of BL clinically manifesting as sepsis in an immunocompetent young adult male. A quick diagnosis with bone marrow biopsy leads to starting treatment with IV chemotherapy. Unfortunately, our patient succumbed to the disease. It is necessary to consider malignancies as one of the main differential diagnosis to facilitate the correct diagnosis and prompt treatment.

Keywords: Burkitt lymphoma; Non-Hodgkin lymphoma; Hyper-CVAD; Sepsis; Lactic acidosis

Abbreviations: BL: Burkitt Lymphoma; IVDU: Intravenous Drug Use; DIC: Disseminated Intravascular Coagulation; TTE: Trans Thoracic Echocardiogram; ID: Infectious Disease; VBG: Venous Blood Gas; CRP: C-Reactive Protein; UDS: Urine Drug Screen; UA: Urine Analysis; BUN: Blood Urea Nitrogen; VDRL: Venereal Disease Research Laboratory; PPD: Purified Protein Derivative; LDH: Lactate Dehydrogenase; PT: Prothrombin Time; INR: International Normalized Ratio

\section{Introduction}

Burkitt Lymphoma (BL) is a rare and highly aggressive B cell neoplasm comprises 30 percent of non-endemic pediatric lymphomas, but less than 1 percent of adult non-Hodgkin lymphomas [1,2]. It is endemic in Africa and sporadic in other parts of the world and is usually diagnosed in children and young adults but rarely in middleaged adults [3]. Patients with BL present with rapidly growing tumor masses and often have evidence of tumor lysis, however sometimes they can present in unusual ways. We present a case of a 29-year old male who presented with tachycardia, persistent lactic acidosis and severe thrombocytopenia. Given his history of IVDU, the initial differential for the patient included sepsis from bacteremia and DIC. The acidosis was so severe and persistent that the patient required intubation from respiratory fatigue and even required dialysis. In addition, despite being on broad spectrum antibiotics he continued to have fevers.

After his workup did not support any of the more common causes of these symptoms, such as negative cultures, further investigation with bone marrow biopsy revealed Burkitt lymphoma. He was started on aggressive treatment with hyper-CVAD alternating with high dose Methotrexate and high dose Cytarabine. Unfortunately, his malignancy was so aggressive and included CNS involvement which leads to tracheostomy placement, paralysis, and eventually death. This is an example of a rare condition with an even more rare presentation. This diagnosis was not reached until many more common diagnoses were ruled out. It is an example of a case where a broad differential is needed to secure the correct diagnosis.

$\mathrm{BL}$ is caused by the translocation and subsequent dysregulation of the c-Myc gene on chromosome 8 [4]. There are 3 distinct clinical forms of Burkitt lymphoma: Endemic (African), Sporadic (nonendemic), and Immunodeficiency Associated [5]. Although these forms differ in their clinical presentation and their epidemiology, they share the same aggressive clinic behavior and are histological identical [6]. The sporadic form is the most common in USA and is identified in this case and represents $30 \%$ of pediatric non-endemic lymphomas and less than $1 \%$ of adult non-Hodgkin lymphomas [7]. However; the clinical presentation of Burkitt's lymphoma will differ depending upon the specific variant. In endemic areas, it usually involves the facial bones, particularly the jaw, maxilla, and orbit, especially in young children. This variant has been associated with Epstein-Barr Virus (EBV) infection, as well as frequent concomitant malaria infection [8]. In comparison, the sporadic form tends to present in the lymphoid tissues of the gut, often presenting as masses 
in the terminal ileum, or even with massive abdominal involvement. Bone marrow involvement is commonly seen in progressive disease [9]. EBV involvement is reported in around $15-30 \%$ of cases [10]. The immunodeficient form is often associated with HIV infection, and may also be seen in post-transplant patients who are chronically immunosuppressed [9].

\section{Case Presentation}

A 29-year old white male presented with bilateral hip and back pain. His past medical history was significant for IVDU. Initially, the symptoms started several weeks ago prior to admission when he began having left hip pain which progressively became worse. He stated the pain spread to involve both hips and he rated it (10/10) and described it as sharp. Any type of movement makes the pain worse. He states that the pain progressed to involve his lower back and described it as throbbing. He also stated that he is having some left lower face tingling. Otherwise he denies any numbness and tingling in lower extremities. He tried taking over the counter Aleve which did not alleviate his pain. Aleve and Ibuprofen were also ineffective at treating the pain. He denies any sick contracts, recent IVDU, including injection into his hip. He did not take any Tylenol to treat the pain. He denies saddle paresthesia or bowel or bladder incontinence. He reports remote IVDU use ( 2 years ago). He went to the outside facility. In the ED he was to found to have a lactic acidosis, thrombocytopenia, leukocytosis, as well as toxology screen positive for suboxone and oxycodone. He was given Vancomycin and Cefepime and was admitted. He underwent multiple imaging modalities including CT brain, CT angiogram head and neck, MRI brain/thoracic spine/hip and pelvis which were unremarkable aside from demonstrating a disc bulge at L1 and L3 and nonspecific bilateral muscle edema (on MRI of pelvis and hip) and a lateral acetabular tear. The patient had a TTE performed that was concerning for a subacute/healed endocarditis. The patient was seen by their ID physician who had changed the patient to Vancomycin, Doxycycline and Imipenem. During the course of the patient's stay his platelets have been trending down with no reported etiology.

He believed that there were times in the recent past where he felt he was warm but never took his temperature. He denied any weight loss, chills, incontinence, sputum production, hemoptysis, and cough. The patient started using IV drugs at age 18. His drug use has included the use of methamphetamine, morphine, cocaine and many other drugs. UDS on admission was positive for Buprenorphine, Benzodiazepine and Opiates. He has had PPDs in the past, his last was approximately 2 years ago, which was negative.

On arrival to Ruby Memorial Hospital, patient was afebrile at 36.9, BP 162/88 mmHg and HR $131 \mathrm{bpm}$. Acutely ill appearing, in acute distress, thin, mucous membranes dry, tachycardia, regular rhythm, no murmurs, no respiratory distress, lungs were clear to auscultation, abdomen with voluntary guarding, diffusely TTP, neurologically intact. Labs were revealed a WBC-10.7; Bands-7; Neutrophils-37; Lymphocytes-46; Monocytes-4; Eosinophils-2; Basophils-2; Hemoglobin-10.7; Platelets-19. Lactic Acid was 11.1. On VBG his pH was 7.32, $\mathrm{pCO}_{2}-34, \mathrm{Na}-133$; K-4.1; Cl-96, $\mathrm{CO}_{2}-15$, AG-22; Ca-9.7; Glucose-45; BUN-16, Cr-0.69, CRP-253, ESR-26, UA: Ketones; UDS: Suboxone and Oxycodone, LDH was too high to be detected, Hepatitis B, C, and HIV serologies negative, Albumin 3.2; Alkaline phosphate 255; AST-267; ALT-112; Total bilirubin-1.3, Direct bilirubin-0.7, PT16.7; INR-1.60; Fibrinogen-311. A chest X-ray, PA and lateral showed no focal infiltrates and an HIV antibody screen was negative, as was a later PCR. CT Chest abdomen and pelvis showed splenomegaly, otherwise within normal limits. Patient was admitted with sepsis suspicion for endocarditis.
After admission, the patient spiked episodic fevers as high as $38.8^{\circ} \mathrm{C}$. He was transferred to ICU service a day later. Since transfer, his lactate had increased from 11.1 to 13.2 and he became tachypneic. ID was consulted and recommended starting him on broad spectrum antibiotics, he was also started on a sodium bicarbonate drip, and he eventually needed intubation for respiratory compromise. He needed emergent dialysis for overwhelming acidosis. TTE with pericardial effusion, no vegetations. MRI brain was within normal limits. Hematology/Oncology was consulted for thrombocytopenia. Initially they were concerned about DIC given his history of IVDU.

Blood and urine cultures were drawn on admission. The following day, the patient continued to have episodic fevers and he also became hypertensive with a BP 190/90. The blood cultures drawn on admission showed no growth in four days. Further workup including ADAMTS13 did not reveal cause of persistent thrombocytopenia. A peripheral smear was performed which showed schistocytes, normochromic normocytic anemia, severe thrombocytopenia, banded neutrophils, myelocytes, RBC inclusion bodies (Figure 1).

Bone marrow was biopsy performed 4 days after admission; the results were consistent with Burkitt Lymphoma findings with the pathognomonic "starry sky" appearance (Figure 2). It showed a hypercellular marrow (greater than $95 \%$ cellularity), flow cytometry was CD10-positive B-Cell lymphoma (76\%), and karyotype: 8;22 translocation.

Throughout his hospital course, patient continued to experience episodic fevers as well as uncontrolled hypertension. Patient also became confused and would regularly only be oriented to person and/ or place. An ophthalmologic exam revealed papilledema. Multiple escalations of his blood pressure regimen failed to bring his pressures within reasonable limits. Patient underwent a traumatic lumbar

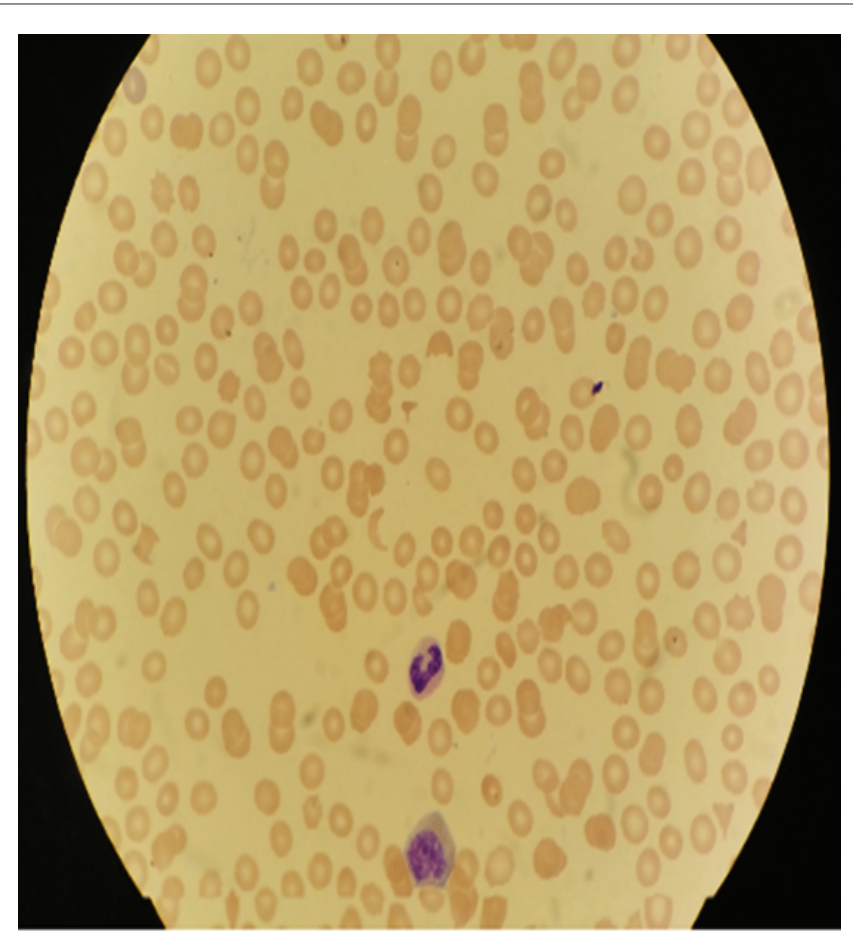

Figure 1: Peripheral smear showed schistocytes, normochromic normocytic anemia, severe thrombocytopenia, banded neutrophils, myelocytes, RBC inclusion bodies. 


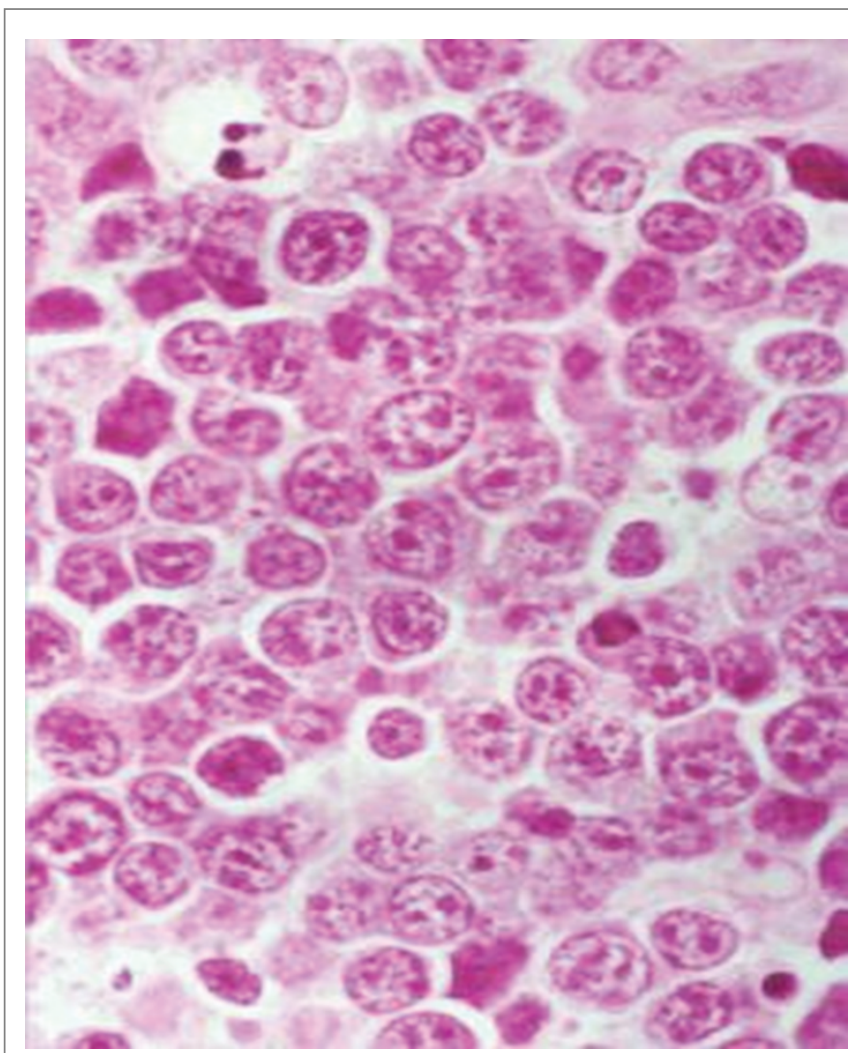

Figure 2: Under the microscope, the hallmark of Burkitt lymphoma is the presence of a "starry sky", imparted by scattered macrophages phagocytizing cell debris and apoptotic cells.

puncture. Opening pressure was $310 \mathrm{mmHg}$ and CSF studies showed glucose of $103 \mathrm{mg} / \mathrm{dL}$, protein of $200 \mathrm{mg} / \mathrm{dL}$, Clotted RBCs, WBCs with 95\% PMNs (Polymorpho Nuclear Leukocytes) and 3\% lymphocytes. Gram stain did not show any organisms and cultures were initiated. CSF flow cytometry at first was non-diagnostic. Additional CSF workup including tuberculosis PCR, VDRL, EBV PCR, CMV PCR, enterovirus, HSV1/HSV2 PCR, and cryptococcal antigen were negative. Blastomycosis serum antibody and histoplasmosis urine antigen were also negative.

After his diagnosis was made, he was started on a cycle of hyper CVAD (Cyclophosphamide, Vincristine, Doxorubicin, and Dexamethasone). Unfortunately, soon after he went into respiratory distress and required BiPAP. He had an echocardiogram which did not show a reduced ejection fraction. His respiratory status continued to worsen and he needed to be intubated. Eventually, he developed critical illness neuropathy and required a tracheostomy placement. His mental status worsened and he was found to have leptomeningeal involvement and required several lumbar punctures. Flow cytometry on repeat spinal tap showed an atypical lymphoid cell that was suspicious for malignancy. He was then started on intrathecal Methotrexate. He later developed paralysis and apnea and was eventually made comfort care and passed as a result of his lymphoma.

\section{Discussion and Conclusion}

Burkitt lymphoma is an extremely rapidly growing tumor with a high sensitivity to chemotherapy but in which drug resistance can develop quickly [9]. Although Burkitt lymphoma is one of the most aggressive types of cancer it is also one of the most curable [7]. Chemotherapy is the mainstay of treatment for Burkitt lymphoma. Treatment of limited stage Burkitt lymphomas is usually very successful in children, with a cure rate of over $90 \%$. For more advanced Burkitt lymphoma cure rate ranges from about $80 \%$ to $90 \%$. However, compared to adults with low risk the five-year survival is $70 \%$ and high risk cases the survival is less than $30 \%$ [4].

The staging workup must be expedited, because Burkitt tumor grows rapidly, causing life-threatening complications. BL is classified as a highly aggressive peripheral B cell tumor and demonstrating the highest proliferation rate of any neoplasms in humans, with a potential doubling time of 24 hour and a growth fraction of nearly $100 \%$ [11]. Sudden and unexpected development of life threatening complications such as airway and abdominal obstruction and acute renal failure has been reported with Burkitt lymphoma in the literature [12]. The diagnosis of Burkitt lymphoma is made by obtaining a biopsy of the tumor mass for histopathology, immunochemistry, and flow cytometry cytogenetic studies to identify c-Myc mutation will aid in the diagnosis. The most suggestive lymph nodes should be selected for excisional biopsy. c-Myc translocation is a characteristic of Burkitt lymphomas. Approximately $80 \%$ of Burkitt lymphoma cases have the $\mathrm{t}(8 ; 12)$ translocations. An increase in $\mathrm{c}-\mathrm{Myc}$ expression results from the translocation. This c-Myc dysregulation seems to be the defining abnormality that eventuates into BL $[13,14]$. They are recognized as small non-cleaved cell lymphomas displaying a starry sky appearance due to the high rate of proliferation and spontaneous cell death (Figure 2).

This case presents a very rare type of presentation of a very rare type of lymphoma in adults. Usually when a young patient like in our present study with fever we think of sepsis. This is an important case because his initial presentation was similar to sepsis and his history of IVDU led us towards a diagnosis of endocarditis. Sepsis will always remain high on the differential diagnosis, however if they do not respond appropriate antibiotics and if cultures are negative other diagnosis need to be considered. One unique presentation that our patient had was severe lactic acidosis and LDH which was not mentioned in other case presentation. A possible explanation could be due to high tumor burden seen in BL similar to a tumor lysis syndrome presentation. A broad differential with alternative diagnoses must be considered in patients who fail to improve with appropriate therapy. In literature review, most cases of BL do not present with lactic acidosis and it is considered a rare complication of BL with only 26 previous reports [15]. However, with those cases that present with lactic acidosis, the mortality rate is at $73 \%$ at 1 month [15]. A possible explanation is that with aggressive lymphoma lactic acidosis is common due to overproduction of lactic acid by rapidly dividing tumor similar to what is seen in BL.

\section{References}

1. Husain M, Grunebaum E, Naqvi A, Atkinson A, Ngan BY, et al. (2007) Burkitt's lymphoma in a patient with adenosine deaminase deficiency-severe combined immunodeficiency treated with polyethylene glycol-adenosine deaminase. J Pediatr 151: 93-95.

2. Wright DH (1999) What is Burkitt's lymphoma and when is it endemic? Blood 93: 758-759.

3. Sangeeta Patankar, Poornima Venkatraman, Gokul Sridharan, Shubhada Kane (2015) Burkitt's lymphoma of maxillary gingiva: A case report. World J Clin Cases 3: 1011-1016.

4. Boerma EG, van Imhoff GW, Appel IM, Veeger NJ, Kluin PM, et al. (2004) Gender and age-related differences in Burkitt lymphoma-epidemiological and clinical data from The Netherlands. Eur J Cancer 40: 2781-2787. 
5. Leoncini L, Raphael M, Stein H, Harris NL, Jaffe ES, et al. (2008) Burkitt lymphoma. In: Swerdlow SH, Campo E, Harris NL, Pileri SA, Jaffe ES, et al. (eds) WHO classification of tumours of haematopoietic and lymphoid tissues. $4^{\text {th }}$ Edition, IARC Press, France 262-264.

6. Molyneux EM, Rochford R, Griffin B, Newton R, Jackson G, et al. (2012) Burkitt's lymphoma. Lancet 379: 1234-1244.

7. Gong JZ, Stenzel TT, Bennett ER, Lagoo AS, Dunphy $\mathrm{CH}$, et al. (2003) Burkitt lymphoma arising in organ transplant recipients: a clinicopathologic study of five cases. Am J Surg Pathol 27: 818-827.

8. McNally RJ, Parker L (2006) Environmental factors and childhood acute leukemias and lymphomas. Leuk Lymphoma 47: 583-598.

9. Khaled M Musallam, Ali T Taher, Ali I Shamseddine (2008) Burkitt's lymphoma of the colon and bronchi: three case report. Cases J 1: 15.

10. Burmeister T, Schwartz S, Horst HA, Rieder H, Gokbuget N, et al. (2005) Molecular heterogeneity of sporadic adult Burkitt type leukemia/lymphoma as revealed by PCR and cytogenetics: correlation with morphology, immunology and clinical features. Leukemia 19: 1391-1398.
11. Kemp S, Gallagher G, Kabani S, Noonan V, O'Hara C (2008) Oral nonHodgkin's lymphoma: review of the literature and World Health Organization classification with reference to 40 cases. Oral Surg Oral Med Oral Pathol Oral Radiol Endod 105: 194-201.

12. Jan A, Vora K, Sandor GK (2005) Sporadic Burkitt's lymphoma of the jaws: the essentials of prompt life-saving referral and management. J Can Dent Assoc 71: 165-168.

13. Neville BW, Damm DD, Allen CM, Bouquot JE (2009) Soft tissue tumors. In: Neville BW (eds) Oral and Maxillofacial Pathology. $3^{\text {rd }}$ Edition, Elsevier 557-559.

14. Yustein JT, Dang CV (2007) Biology and treatment of Burkitt's lymphoma. Curr Opin Hematol 14: 375-381.

15. Glasheen JJ, Sorensen M (2005) Burkitt's lymphoma presenting with lactic acidosis and hypoglycemia-a case presentation. Leuk Lymphoma 46: 281-283. 